\title{
Evaluation of the Clinical Profile and Malignancies in Children With Neurofibromatosis Type 1
}

Nihal Şahin ( $\nabla$ nihal_sahin41@hotmail.com )

Kocaeli University: Kocaeli Universitesi https://orcid.org/0000-0002-2122-6952

\section{Ugur Demirsoy}

Kocaeli Üniversitesi: Kocaeli Universitesi

\section{Funda Corapcioglu}

Kocaeli University: Kocaeli Universitesi

\section{Research Article}

Keywords: Children, low grade glial tumors, neurocutaneous syndromes, neurofibromatosis

Posted Date: March 25th, 2021

DOI: https://doi.org/10.21203/rs.3.rs-342639/v1

License: (1) This work is licensed under a Creative Commons Attribution 4.0 International License. Read Full License 


\section{Abstract}

\section{Purpose:}

Neurofibromatosis type 1 (NF 1) is a significant disease as it is one of the most common autosomal dominant disorders in childhood. Several systems are affected due to significant progression. This study aimed to analyze the clinical findings in children with NF 1 and investigate the characteristics of those with malignancy.

\section{Methods:}

Medical records of 55 children with NF 1 that were followed up for ten years (2004-2015) in our center were analyzed. We assessed clinical and demographical characteristics of patients, presence NF 1 diagnostic criteria, NF 1 related complications, and malignancies. The patients without malignancy are classified in group 1 while patients with malignancy are in group 2 .

\section{Results:}

The mean age was $7.68 \pm 4.65$ years. Female gender was dominant in both groups. Café au lait spots were present in all patients. Axillary-inguinal freckling was observed in $76.4 \%$ of patients, followed by neurofibromas in $30.9 \%$, Lisch nodules in $29.1 \%$, bone dysplasia in $14.5 \%$, optic gliomas (OG) in $23.6 \%$, and a history of first degree relative with NF 1 in $63.6 \%$. Central nervous system (CNS) tumors were present in $40 \%$. Tumors beyond CNS were acute myeloid leukemia and schwannoma. None of the diagnostic criteria was a risk factor for malignancy. Having $>3$ criteria was the risk factor for malignancy in NF-1 (OR:5.891, Cl 95\%: 1.676-20.705, p=0.006).

\section{Conclusions:}

The major problem is malignancies in NF -1 patients. There are no clearly defined risk factors predicting malignancies in NF-1 at present. However, we found the risk of malignancy higher in patients with more diagnostic criteria.

\section{Introduction}

Neurofibromatosis type 1 (NF-1) is one of the common neurocutaneous diseases. Recent studies report a birth incidence of $1 / 2000$ and prevalence of $1 / 4000$ [1-3]. It has a broad spectrum of clinical effects on several organ systems [4]. NF-1 has an autosomal dominant pattern of inheritance, while half of the patients have a de novo mutation. It is caused by mutations of the NF-1 gene located in 17q11.2. NF-1 gene encodes neurofibromin which is one of the tumor suppressor proteins [5]. This mutation provokes proliferation and tumorigenesis in various tissues, especially in neurocutaneous tissue. The most common features in NF-1 are hyperpigmented macules called café-au-lait macules (CALMs), Lisch nodules, and neurofibromas. The malignancy rate is high in patients with NF-1. Central nervous system (CNS) tumors, malignant peripheral nerve sheath tumor (MPNST), leukemias and rhabdomyosarcoma 
(RMS) are the mostly reported malignancies in NF-1 [4]. The life expectancy is 10-15 years lower in NF-1 patients compared to healthy population [6]. Clinical findings vary among affected individuals due to highly variable clinical expressivity. Manifestations of NF-1 increase with age making clinical picture worse at older ages [7-9].

In this study, we aimed to determine the clinical features of NF-1 patients and the remarkable characteristics of NF 1 patients with malignancy and evaluate the treatment and follow-up outcomes.

\section{Methods}

\section{Patients data}

We scanned electronic medical records of NF-1 patients followed at Kocaeli University Pediatric Oncology Department in years 2004-2015 We reached files of 88 patients that were diagnosed with NF-1. Fifty-five patients who fulfilled the inclusion criteria were enrolled. Inclusion criteria were age under 18 years, a minimum follow-up time of three months in our center, meeting the National Institutes of Health (NIH) NF1 diagnostic criteria [10], and having full access to medical records regarding diagnosis and treatment.

Demographic findings, diagnostic criteria, clinical findings other than diagnostic criteria, malignancy, and family history were evaluated. We grouped the patients considering presence of malignancy. The patients without malignancy were in group 1 ; patients with malignancy were in group 2 . We evaluated treatment modalities, treatment response, and prognosis of the disease in group 2.

\section{Ethics and consent to participate declarations}

All patients/parents signed the consent form and accepted use of their medical records for scientific research. The Ethics Committee of Kocaeli University had approved this study (KOU KAEK 14/98).

\section{Statistical analysis}

We used IBM SPSS 20.0 (SPSS Inc., Chicago, IL, USA) for statistical analysis. Normality tests were performed for continuous variables. Data were presented as mean \pm standard deviation for normally distributed continuous variables, median (25-75 percent) for abnormally distributed continuous variables, and proportions for categorical variables. Continuous variables showing normal distribution were compared between groups using Student's t-test, and abnormally distributed variables were compared using the Mann-Whitney $U$ test. Categorical variables were compared using the Chi-square test. Univariate binary logistic regression analysis was performed to identify the risk factors for malignancy in NF 1. Odds ratios were calculated with $95 \%$ confidence intervals. A p-value of less than 0.05 was considered statistically significant.

\section{Results}


Thirty-two patients $(58.2 \%)$ were female. The mean age at diagnosis was $7.68 \pm 4.65$ years; the median follow-up duration was $2(0.08-11.50)$ years. The mean age at diagnosis of malignancy was $8.90 \pm 4.22$ years. The demographic and clinical characteristics of both groups were summarized in table 1.

\section{The diagnostic criteria of patients}

All patients had CALMs. Axillary/groin freckling followed CALMs in 42 (76.4\%) patients. Seventeen patients $(30.9 \%)$ had neurofibromas. Cutaneous neurofibromas and plexiform neurofibroma were observed in $11(20 \%)$ and 10 (18.2\%) patients, respectively. Eight (14.5\%) patients had bone dysplasia. Congenital tibial pseudoarthrosis was observed in $3(5.4 \%)$ patients; while congenital tibial dysplasia in 2 (3.6\%) patients; sphenoid wing dysplasia in $1(1.8 \%)$ patient; ulnar dysplasia was in $1(1.8 \%)$ patient and humerus dysplasia in $1(1.8 \%)$ patient. The other diagnostic criteria were showed in table 1.

The frequency of diagnostic criteria was not different between 0-1 age-year, 2-6 age-year and $\geq 7$ age-year groups. No patients had Lisch nodules or bone dysplasia in the 0-1 age-year group. The number of criteria did not correlate with the age at diagnosis NF-1 $(r(55)=0.34 ; p=0.80)$.

\section{The clinical findings out of the diagnostic criteria}

Weight and height percentiles were below the $3^{\text {rd }}$ percentile in 19 (34.5\%) and 11 (20\%) patients, respectively. Head circumference percentile was above the $90^{\text {th }}$ in $18(32.7 \%)$ patients and was below the $3^{\text {rd }}$ in $1(1.8 \%)$ patient.

Three (5.5\%) patients had delayed puberty, and 2 (3.6\%) patients had precocious puberty. All patients with delayed puberty had malignancy. These tumors were cerebellar astrocytoma in one patient, cerebral hemispheric glioma in one, and brainstem and cerebral hemispheric glioma in one.

Cranial magnetic resonance imaging (MRI) was performed in $51(92.7 \%)$ patients, and $43(78.2 \%)$ of these patients had abnormalities on MRI . The cranial MRI abnormality was unidentified bright objects (UBO) in 40 (72.2\%) patients. Other cranial MRI abnormalities except for UBO and malignancy were present in $12(21.8 \%)$ patients. Ten (18.2\%) patients had an abdominal abnormality, 4 (7.3\%) patients had a urinary abnormality on ultrasonography; 2 (3.6\%) patients had an echocardiographic abnormality and, $7(12.7 \%)$ patients had endocrinologic disorders (Table 2 ).

We compared patients with and without UBO regarding seizures, mental retardation, and cranial malignancies, and we did not find a significant difference (Table 3). Eleven (20\%) patients had seizures. The cause of seizures was febrile convulsion and epilepsy in $5(9.1 \%)$ and $6(10.9 \%)$ patients, respectively. Mental retardation was mild in $19(34.5 \%)$ patients, moderate in $6(10.9 \%)$ patients and severe in 2 (3.6\%) patients. Other neurological problems were headache due to CNS tumor in 2 (3.6\%) patients, urinary and bowel incontinence in $1(1.8 \%)$, and hemiplegia in $1(1.8 \%)$ patient due to plexiform neurofibroma. 


\section{Malignancies of the patients}

Malignancy was detected in $23(41.8 \%)$ patients. Fourteen (60.9\%) of these patients were diagnosed with NF-1 during workup for malignancy. The longest duration between diagnosis of NF-1 and malignancy was three years. Three (60\%) patients in the 0-1 age group, 4 (25\%) patients in the 2-6 age group, 16 $(47.1 \%)$ patients in the $\geq 7$ age group had malignancy $(p=0.257)$. We evaluated the diagnostic criteria as possible risk factors for malignancy with univariate logistic regression analysis. None of the diagnostic criteria was a risk factor for malignancy in NF-1 (Table 4). We compared the number of diagnostic criteria (optic glioma criteria was excluded) between patients with and without malignancy. The patients were grouped in three groups; patients with less than 3 criteria, with 3 criteria and more than 3 criteria. Eleven $(20.6 \%)$ of patients with less than or with 3 criteria and $12(76.2 \%)$ of patients with more than 3 criteria had malignancy $(p=0.004)$. In univariate regression analysis, having more than 3 criteria was the risk factor for malignancy in NF-1 (OR:5.891, Cl 95\%: 1.676-20.705, $p=0.006)$.

There was at least one CNS tumor in $22(40 \%)$ patients. Two (3.5\%) patients had malignancies other than CNS tumors. Optic glioma in $13(23.6 \%)$ patients was the most prevalent tumor (Table 5). Seven (30.4\%) patients with tumors were followed without treatment. Sixteen (69.6\%) patients were treated for progressive disease and received chemotherapy (CTX). In 2 patients who received CTX, the tumor was excised before CTX. One of these patients had glioma in the hypothalamus, and the other had glioma in the right temporal lobe. Glioblastoma multiforme (GBM) was detected on the biopsy of the patient with temporal lobe glioma. Two patients with GBM and brainstem glioma received radiotherapy (XRT).

Eight (50\%) of patients who received CTX were given carboplatin-vincristine (CV) combination.

Carboplatin-vincristine combination was switched to temozolomide in two of these patients (12.5\%) due to progressive disease. Five (31.2\%) patients with low-grade glial tumors received temozolomide as a primary CTX protocol. In one patient with acute myeloid leukemia, CTX induction was applied before bone marrow transplantation. Only one patient showed an allergic reaction to carboplatin at the last cycle, and the treatment was stopped. Thirteen (81.3\%) patients finished the treatment. Three $(18.7 \%)$ patients left the treatment.

In the patients group with malignancy, 9 (39.1\%) had stable disease, $4(17.4 \%)$ had a partial response, and $3(13 \%)$ had progressive disease. Seven patients did not follow up. One of the patients with progressive disease had GBM, and received temozolomide and XRT after tumor excision. However, the patient died due to progression of the tumor. The characteristics of patients with malignancy were shown in supplemental Table 1.

\section{Discussion}

Our study showed that the clinical heterogeneity of NF-1 in children is as broad as in adults, and the risk of malignancy increases in patients with more than three NIH criteria (excluding optic glioma). 
Neurofibromatosis type 1 occurs in childhood and is one of the most common autosomal dominant diseases [11-13]. Since the disease penetrance is $100 \%$, the number and severity of clinical symptoms increase with age. Our results, that, more than half of patients were seven years old and had three diagnostic criteria, were consistent with this fact. However, we did not find a linear correlation between the age of diagnosis and the number of criteria. Café-au-lait macules were one of the primary diagnostic criteria. Café-au-lait macules start to occur after birth, and both, the diameter and the number of CALMs increase with age $[14,15]$. All of our patients had greater than/equal to 6 CALMs. Previously studies detected cutaneous neurofibroma in more than $80 \%$ of patients and plexiform neurofibroma in $30-50 \%$ of patients $[15,16]$. Neurofibromas occur in adolescence and after $[15,16]$. Some patients in our study were in adolescence or older. Therefore, the frequency of neurofibroma was low in our study. Another classical feature of NF-1 is the dysplasia of long bones in infants [9]. The frequency of bone dysplasia in our patients was similar to literature.

In NF-1, several features other than malignancies vary by age and interest to organ systems [16]. Although our study was held in a pediatric group, we detected several manifestations in almost all organ systems. Unidentified bright object, mental retardation, and scoliosis were common manifestations of our patients which are not classified in diagnostic criteria.

In our study, unidentified bright object was more common than axillary or inguinal freckling, even though UBO is not a diagnostic criterion. Some researchers suggested that UBO can be used as another diagnostic criteria [17-21]. Our result supports this attitude. Although, an association between cognitive disorders and UBO was detected in previous studies [22], we did not find a relationship between mental retardation and UBO. Also, malignancies of CNS and seizures were not significant in our patients with UBO.

The most common malignancies are intracranial tumors in NF1. Optic glioma is the primary intracranial tumor in NF-1. The frequency of optic glioma was reported as $15-20 \%$ in children with NF-1 [4]. Varan et al. [23] found intracranial tumors other than optic glioma in $2.3 \%$ of patients with NF-1. Almost half of our patients had a malignancy, and we detected optic glioma in $23.6 \%$ of them. Besides, the frequency of other intracranial tumors was ten times higher in our study. We think that these results were provided by the help of detailed evaluation of NF-1 patients referred to our pediatric oncology clinic with an intracranial tumor. Schwannoma, meningioma, and acute myeloid leukemia were malignancies other than gliomas in our patients.

A study which evaluated CV efficiency on progressive low-grade glioma showed that event-free survival and tumor response rates were superior in children with NF-1 compared to children without NF-1 [24]. Temozolomide did not have a superior effect than $\mathrm{CV}$ in regard to survival of patients with low-grade glioma in past studies. However, temozolomide is increasingly preferred due to its better tolerance and easy administration [25]. We used CV in 8 and temozolomide in 5 low-grade glioma patients as primer CTX protocol. Carboplatin-vincristine was switched to temozolomide in one patient due to progressive disease We added RTX to CTX in two patients. One of these had GBM, and the other had brainstem 
glioma. The patient with GBM died due to progressive disease. High-grade CNS tumors were reported in a few patients with NF-1, and these patients had a poor prognosis, too [26].

In the recent years, several studies about risk factors of glioma formation and progression in NF-1 were reported. Various factors, such as the germline NF1 gene mutation, patient age, patient gender, background genomics (ethnicity/race), co-existing atopic conditions (eczema, asthma), were investigated [27]. Risk factors related to vision loss were female gender, age less than two years, and posterior involvement in optic glioma with NF-1, as in optic glioma without NF-1 [28-30]. However, optic glioma incidence in NF1 is similar in male and female gender [31,32]. Tabata et al. [16] did a cluster analysis in adults with NF 1 and found positive correlations between spinal neurofibromas and optic gliomas; between optic gliomas and sphenoid wing dysplasia. Also, they reported that increasing cutaneous neurofibromas was a risk factor for MPNST [16]. In our study, gender and age were unremarkable in patients with malignancy. We could not find any NIH diagnostic criteria as a risk factor in the univariate analysis of malignancy-risk factors. However, we found that having more than three diagnostic criteria except optic glioma increased the risk of malignancy six times.

In conclusion, children with NF-1 have clinical heterogeneity similar to adult patients. Malignancies are the most crucial factor in mortality and morbidity in NF-1. Risk factors for developing malignancy in NF-1 are still unclear. However, we suggest being vigilant about potential malignancy in patients with more diagnostic criteria.

\section{Declarations}

Funding: No funds, grants, or other support was received.

Conflicts of interest: None of the authors have any conflicts of interest.

Authors' contributions: All authors contributed to the study conception and design and had an essential part in caring for the patients whose data was used in this manuscript. Data collection and analysis were performed by NS. The first draft of the manuscript was written by NS and the final draft edited and approved by UD and FC.

Ethics approval: The Ethics Committee of Kocaeli University had approved the study (KOU KAEK 14/98).

Consent to participate: Informed consent was obtained from legal guardians.

Data availability: The datasets generated during and/or analyzed during the current study are available from the corresponding author on reasonable request.

\section{References}

1. Rasmussen SA, Friedman JM (2000) NF1 gene and neurofibromatosis 1. Am J Epidemiol 151:33-40 
2. Uusitalo E, Leppävirta J, Koffert A, et al (2015) Incidence and mortality of neurofibromatosis: A total population study in Finland. J Invest Dermatol 135:904-906. https://doi.org/10.1038/jid.2014.465

3. Kallionpää RA, Uusitalo E, Leppävirta J, et al (2018) Prevalence of neurofibromatosis type 1 in the Finnish population. Genet Med 20:1082-1086. https://doi.org/10.1038/gim.2017.215

4. Bergqvist C, Servy A, Valeyrie-Allanore L, et al (2020) Neurofibromatosis 1 French national guidelines based on an extensive literature review since 1966. Orphanet J Rare Dis 15:37

5. Le C, Bedocs PM (2020) Neurofibromatosis. StatPearls Publishing

6. Duong TA, Sbidian E, Valeyrie-Allanore L, et al (2011) Mortality associated with neurofibromatosis 1 : A cohort study of 1895 patients in 1980-2006 in France. Orphanet J Rare Dis 6:18. https://doi.org/10.1186/1750-1172-6-18

7. Williams VC, Lucas J, Babcock MA, et al (2009) Neurofibromatosis type 1 revisited. Pediatrics 123:124-133

8. DeBella K, Szudek J, Friedman JM (2000) Use of the National Institutes of Health criteria for diagnosis of neurofibromatosis 1 in children. Pediatrics 105:608-614. https://doi.org/10.1542/peds.105.3.608

9. Boulanger JM, Larbrisseau A (2005) Neurofibromatosis type 1 in a pediatric population: Ste-Justine's experience. Can J Neurol Sci 32:225-231

10. (1988) Neurofibromatosis: Conference Statement. Arch Neurol 45:575-578. https://doi.org/10.1001/archneur.1988.00520290115023

11. Plon S, Malkin D (2006) Childhood cancer and heredity. In: Pizzo P, Poplack D (eds) Principles and Practice of Pediatric Oncology, 5th ed. Lippincott-Roven, Philadelphia, PA, pp 14-37

12. Gutmann DH (1997) The Diagnostic Evaluation and Multidisciplinary Management of Neurofibromatosis 1 and Neurofibromatosis 2. J Am Med Assoc 278:51. https://doi.org/10.1001/jama.1997.03550010065042

13. Friedman JM (2002) Neurofibromatosis 1: Clinical manifestations and diagnostic criteria. J. Child Neurol. 17:548-554

14. Korf BR (1992) Diagnostic outcome in children with multiple cafe au lait spots. Pediatrics 90:924927

15. Ferner RE, Huson SM, Thomas N, et al (2007) Guidelines for the diagnosis and management of individuals with neurofibromatosis. J Med Genet 44:81-88

16. Tabata MM, Li S, Knight P, et al (2020) Phenotypic heterogeneity of neurofibromatosis type 1 in a large international registry. JCl Insight 5:136262. https://doi.org/10.1172/jci.insight.136262

17. Szudek J, Friedman JM (2002) Unidentified bright objects associated with features of neurofibromatosis 1. Pediatr Neurol 27:123-127. https://doi.org/10.1016/S0887-8994(02)00403-4

18. Lopes Ferraz Filho JR, Munis MP, Soares Souza A, et al (2008) Unidentified bright objects on brain MRI in children as a diagnostic criterion for neurofibromatosis type 1. Pediatr Radiol 38:305-310. https://doi.org/10.1007/s00247-007-0712-x 
19. Tadini G, Milani D, Menni F, et al (2014) Is it time to change the neurofibromatosis 1 diagnostic criteria? Eur J Intern Med 25:506-510

20. Curless RG, Siatkowski M, Glaser JS, Shatz NJ (1998) MRI diagnosis of NF-1 in children without cafe-au-lait skin lesions. Pediatr Neurol 18:269-271. https://doi.org/10.1016/S0887-8994(97)001896

21. DeBella K, Poskitt K, Szudek J, Friedman JM (2000) Use of "unidentified bright objects" on MRI for diagnosis of neurofibromatosis 1 in children. Neurology 54:1646-1650. https://doi.org/10.1212/wnl.54.8.1646

22. Farrer MJ, Praticò AD, Montenegro MA, et al (2020) Can the Cognitive Phenotype in Neurofibromatosis Type 1 (NF1) Be Explained by Neuroimaging? A Review. Front Neurol 1:1373. https://doi.org/10.3389/fneur.2019.01373

23. Varan A, Şen H, Aydin B, et al (2016) Neurofibromatosis type 1 and malignancy in childhood. Clin Genet 89:341-345. https://doi.org/10.1111/cge.12625

24. Ater JL, Xia C, Mazewski CM, et al (2016) Nonrandomized comparison of neurofibromatosis type 1 and non-neurofibromatosis type 1 children who received carboplatin and vincristine for progressive low-grade glioma: A report from the Children's Oncology Group. Cancer 122:1928-1936. https://doi.org/10.1002/cncr.29987

25. Van Den Bent MJ (2015) Chemotherapy for low-grade glioma: When, for whom, which regimen? Curr Opin Neurol 28:633-638

26. Lobbous M, Bernstock JD, Coffee E, et al (2020) An Update on Neurofibromatosis Type 1-Associated Gliomas. Cancers (Basel) 12:114. https://doi.org/10.3390/cancers12010114

27. Costa ADA, Gutmann DH (2020) Brain tumors in neurofibromatosis type 1. Neuro-Oncology Adv 2:85-97. https://doi.org/10.1093/noajnl/vdz040

28. Fisher MJ, Loguidice M, Gutmann DH, et al (2012) Visual outcomes in children with neurofibromatosis type 1-associated optic pathway glioma following chemotherapy: A multicenter retrospective analysis. Neuro Oncol 14:790-797. https://doi.org/10.1093/neuonc/nos076

29. Liu GT, Brodsky MC, Phillips PC, et al (2004) Optic radiation involvement in optic pathway gliomas in neurofibromatosis. Am J Ophthalmol 137:407-414. https://doi.org/10.1016/j.ajo.2003.09.055

30. Balcer LJ, Liu GT, Heller G, et al (2001) Visual loss in children with neurofibromatosis type 1 and optic pathway gliomas: Relation to tumor location by magnetic resonance imaging. Am J Ophthalmol 131:442-445. https://doi.org/10.1016/S0002-9394(00)00852-7

31. Diggs-Andrews KA, Brown JA, Gianino SM, et al (2014) Sex Is a major determinant of neuronal dysfunction in neurofibromatosis type 1. Ann Neurol 75:309-316. https://doi.org/10.1002/ana.24093

32. Fisher MJ, Loguidice M, Gutmann DH, et al (2014) Gender as a disease modifier in neurofibromatosis type 1 optic pathway glioma. Ann Neurol 75:799-800

\section{Tables}


Table 1: The comparison of clinical characteristics according to the presence of malignancy

\begin{tabular}{|llll|}
\hline Variables & Group 1 & Group 2 & $\mathrm{p}$ \\
\hline Age of diagnosis (year) $^{\mathrm{a}}$ & $7.38 \pm 4.61$ & $8.07 \pm 4.77$ & 0.61 \\
\hline Duration of follow-up (year) $^{\mathrm{b}}$ & $2.00(0.08-11.50)$ & $2.08(0.08-9.90)$ & 0.61 \\
\hline Female & $19(59.4 \%)$ & $13(56.5 \%)$ & 0.83 \\
\hline Cafe au lait spots & $32(100 \%)$ & $23(100 \%)$ & - \\
\hline Neurofibroma & $8(25 \%)$ & $9(39.1 \%)$ & 0.26 \\
\hline Lisch nodules & $7(21.9 \%)$ & $9(39.1 \%)$ & 0.17 \\
\hline Freckling & $22(68.8 \%)$ & $20(87 \%)$ & 0.12 \\
\hline Optic glioma & - & $13(56.5 \%)$ & - \\
\hline Bone dysplasia & $5(15.6 \%)$ & $3(13 \%)$ & 1.00 \\
\hline Neurofibromatosis type 1 in family & $20(62.5 \%)$ & $15(65.2 \%)$ & 0.84 \\
\hline Malignancy in family & $4(12.5 \%)$ & $5(21.7 \%)$ & 0.36 \\
\hline
\end{tabular}

${ }^{a}$ mean $\pm S D,{ }^{b}$ median (minimum-maximum)

Table 2: Abnormalities detected in systemic examinations 


\begin{tabular}{|c|c|}
\hline Abnormalities & $\mathrm{n}$ \\
\hline Skeletal abnormalities other than diagnostic criteria & 20 \\
\hline Scoliosis & 10 \\
\hline Pectus excavatum & 6 \\
\hline Cubitus valgus & 1 \\
\hline Genu valgum & 1 \\
\hline Klindodactyly & 1 \\
\hline $\begin{array}{l}\text { Scoliosis, genu valgum, pectus excavatum, } \\
\text { duplication of finger }\end{array}$ & 1 \\
\hline Abnormalities in cranial imaging other than malignancy and UBO & 12 \\
\hline Hydrocephalus & 4 \\
\hline Asymmetric ventricular volume & 3 \\
\hline Aquaduct stenosis & 2 \\
\hline Arachnoid cyst & 1 \\
\hline Putamen cyst & 1 \\
\hline Wallerian degeneration & 1 \\
\hline Abnormalities in abdominal ultrasonography * & 10 \\
\hline Splenomegaly & 6 \\
\hline Hepatomegaly & 4 \\
\hline Accessory spleen & 3 \\
\hline Endocrinologic abnormalities & 7 \\
\hline Hypothyroidism & 3 \\
\hline Growth hormone deficiency & 2 \\
\hline Type 1 Diabetes mellitus & 1 \\
\hline Panhypopituitarism $†$ & 1 \\
\hline Abnormalities in urinary ultrasonography & 4 \\
\hline Increase in renal parenchyma echo & 1 \\
\hline Decreased kidney size & 1 \\
\hline Hydronephrosis & 1 \\
\hline Nephrolithiasis & 1 \\
\hline
\end{tabular}


Abnormalities in echocardiography

Tricuspid regurgitation

Mitral valve prolapse, mitral regurgitation, tricuspid regurgitation

* Three patients had more than one abdominal ultrasonography pathology

${ }^{\dagger}$ Secondary to hypothalamic glioma resection

Table 3: Evaluation of patients with unidentified bright object

\begin{tabular}{|llll|}
\hline Variables & UBO (+) & UBO (-) & $\mathrm{p}$ \\
& $\mathrm{n}=40(100 \%)$ & $\mathrm{n}=15(100 \%)$ & \\
\hline Mean age (years) & $7.20 \pm 4.03$ & $8.96 \pm 5.97$ & 0.30 \\
\hline Seizures & $8(20 \%)$ & $3(20 \%)$ & 1.00 \\
No seizures & $32(80 \%)$ & $12(80 \%)$ & \\
\hline No mental retardation & $18(45 \%)$ & $10(66.7 \%)$ & 0.08 \\
Mild mental retardation & $15(37.5 \%)$ & $4(26.7 \%)$ & \\
Moderate mental retardation & $5(12.5 \%)$ & $1(6.7 \%)$ & \\
Severe mental retardation & $2(5 \%)$ & $0(0 \%)$ & \\
\hline CNS tumor & $18(45 \%)$ & $4(26.7 \%)$ & 0.35 \\
No CNS tumor & $22(55 \%)$ & $11(73.3 \%)$ & \\
\hline
\end{tabular}

${ }^{*}$ CNS: Central nervous tumor, UBO: Unidentified bright object

Table 4: Evaluation of risk factors for malignancy development with univariate logistic regression analysis 


\begin{tabular}{|llll|}
\hline Variables & $\mathrm{p}$ & $\mathrm{OR}$ & $95 \% \mathrm{Cl}$ \\
\hline Follow-up duration & 0.78 & 0.973 & $0.800-1.182$ \\
\hline Gender & 0.83 & 1.124 & $0.380-3.327$ \\
\hline Neurofibroma & 0.26 & 1.929 & $0.606-6.142$ \\
\hline Freckling & 0.13 & 3.030 & $0.729-12.603$ \\
\hline Bone dysplasia & 0.79 & 0.810 & $0.173-3.792$ \\
\hline Neurofibromatosis type 1 in family & 0.4 & 0.889 & $0.291-2.720$ \\
\hline Malignancy in family & 0.37 & 1.944 & $0.460-8.223$ \\
\hline The number of diagnostic criteria $>3$ & $\mathbf{0 . 0 0 6}$ & $\mathbf{5 . 8 9 1}$ & $\mathbf{1 . 6 7 6 - 2 0 . 7 0 5}$ \\
\hline
\end{tabular}

Table 5: Dispersion of malignancies in patients

\begin{tabular}{|ll|}
\hline Tumors & $\mathrm{n}(\%)$ \\
\hline Central nervous system tumors & $22(40 \%)$ \\
\hline Glial tumors & $22(40 \%)$ \\
Optic glioma & $13(23.6 \%)$ \\
Cerebral hemispheric glioma & $7(12.7 \%)$ \\
Brainstem glioma & $5(9.1 \%)$ \\
Cerebellar astrocytoma & $1(1.8 \%)$ \\
Meningioma & $3(5.4 \%)$ \\
\hline Non-Central nervous system tumors & $2(3.6 \%)$ \\
\hline Schwannoma & $1(1.8 \%)$ \\
Acute myeloid leuchemia & $1(1.8 \%)$ \\
\hline
\end{tabular}

*Five patients had more than one tumor

\section{Supplementary Files}

This is a list of supplementary files associated with this preprint. Click to download. 
- Supplementaltable.docx

Page 14/14 